\title{
DOI https://doi.org/10.30525/978-9934-26-178-7-20
}

\section{THE URGENCY OF THE PROBLEM SYNTHETICALLY REPRODUCED MEDIACONTENT}

\author{
Chemerys H. Yu. \\ PhD, \\ Head of the Department of Design \\ Briantseva H. V. \\ PhD, \\ Assistant Professor at the Department of Design \\ Briantsev O. A. \\ Senior Lecturer at the Department of Design \\ Zaporizhzhia National University \\ Zaporizhzhia, Ukraine
}

The progressive development of information and computer technologies has significantly influenced the transformation of the media landscape, as modern computer graphics tools are used to create photorealistic images (from conventional Photoshopping, to modeling objects for mixed realities AR / VR / XR [4; 5], anamorphic illusions and Virtual Performances), use neural networks and artificial intelligence (NeuroArt; CheepFake, DeepFake, DeepNude; application of CryptoArt; Face Swapping, etc.). The use of synthetically created media content to manipulate public opinion is a threat to the national security of any state. The formation of an appropriate level of critical thinking will ensure the existence of society's immunity to cyber threats, eliminates the negative impact of misinformation on the sustainable development of Ukraine.

To strengthen the fight against misinformation, the European Commission will focus its efforts on revising the Code of Practice on Disinformation in the system of regulation of obligations and accountability of Internet platforms in accordance with the future Digital Services Act. In general, the European Commission aims to introduce tools that would increase the responsibility of the media and the beneficiaries of information campaigns for the dissemination of misinformation. Urgency of the problem is confirmed by a number of normative and legislative documents aimed at regulating measures to ensure national information security and the right to access reliable information. The main requirements for the introduction and development of information, telecommunications and media technologies in Ukraine are contained in the Strategy for the Development of the Information Society in 
Ukraine (2013). In order to counter hybrid threats and spread misinformation in Ukraine approved and implemented the cyber security strategy of Ukraine for 2021-2025 "Safe cyberspace - the key to successful development of the country"; National Security Strategy of Ukraine and the Law of Ukraine "On National Security" (2018). In particular, on July 15, 2021, representatives of state authorities of Ukraine and the delegation of foreign states to the OSCE discussed the fight against misinformation and information security, during which the best world practices of protecting society from the harmful effects of misinformation and other hybrid tools were discussed. In particular, a number of foreign regulations have been formulated, such as the Code of European Union Practice Regarding Disinformation (2018), and the European Commission has adopted a "Joint Framework on Counter-Hybrid Response", European Union response (2016).

In recent years, fake news has become an issue that threatens public discourse, human society and democracy [3, p. 4; 12, p. 16]. Fake news means fake news-style content fabricated to misinform the public $[1 ; 10]$. False information is spread through social networks and easily overcomes any boundaries where it can affect millions of users [8]. This growing popularity of video content emphasizes the need to develop techniques to form critical public thinking to determine the authenticity of media and news, as new technologies allow convincing manipulation of visual information [2]. Today we live in an era characterized by digital disinformation and information warfare, fake information campaigns are being conducted to manipulate public opinion $[2 ; 12 ; 13]$. Recent technological advances have simplified the creation of visual content using "Deepfake" technology, which results in hyperrealistic visual content using face swap that leaves no trace of manipulation $[6$, p. $4-5 ; 11$, p. 256]. Deepfake technology can create, for example, a humorous, pornographic, or political video where someone says something, without the consent of the person whose image and voice are involved [7, p. 108; 9, p. 455-456]. Deepfake technology is particularly dangerous due to its ability to generate counterfeit visual content for political sabotage, fake video evidence in court, terrorist propaganda, blackmail, market manipulation and fake news [11]. Due to the psychological characteristics of a person, after seeing with one's own eyes, trust is instilled, which creates a basis for manipulation of consciousness through misinformation by means of synthetically reproduced mediacontent using Deepfake technology and others.

\section{Refereneces:}

1. Aldwairi M., Alwahedi A. Detecting fake news in social media networks. Procedia Computer Science. 2018. Vol. 141. Pp. 215-222 
2. Anderson K. E. Getting acquainted with social networks and apps: combating fake news on social media. Library Hi Tech News. 2018.

3. Borges L., Martins B., Calado P. Combining similarity features and deep representation learning for stance detection in the context of checking fake news. Journal of Data and Information Quality (JDIQ). 2019. Vol. 11. №. 3. Pp. 1-26.

4. Chemerys H. Reasoning the necessity of study basis of computer design by future bachelors in computer sciences. European Journal of Humanities and Social Sciences. 2019. №. 5. Pp. 52-55.

5. Chemerys H., Vynogradova A., Briantseva H., Sharov S. Strategy for Implementing Immersive Technologies in the Professional Training Process of Future Designers. Journal of Physics: Conference Series. IOP Publishing, 2021. Vol. 1933. №. 1. Pp. 012046.

6. Chawla R. Deepfakes: How a pervert shook the world. International Journal of Advance Research and Development. 2019. Vol. 4. №. 6. Pp. 4-8.

7. Day C. The future of misinformation. Computing in Science \& Engineering. - 2019. Vol. 21. №. 1. Pp. 108-108.

8. Figueira Á., Oliveira L. The current state of fake news: challenges and opportunities. Procedia Computer Science. 2017. Vol. 121. Pp. 817-825.

9. Fletcher J. Deepfakes, artificial intelligence, and some kind of dystopia: The new faces of online post-fact performance. Theatre Journal. 2018. Vol. 70. №. 4. Pp. 455-471.

10. Jang S. M., Kim J. K. Third person effects of fake news: Fake news regulation and media literacy interventions. Computers in human behavior. 2018. Vol. 80. Pp. 295-302.

11. Maras M. H., Alexandrou A. Determining authenticity of video evidence in the age of artificial intelligence and in the wake of deepfake videos. The International Journal of Evidence \& Proof. 2019. Vol. 23. №. 3. Pp. 255-262.

12. Qayyum A. et al. Using blockchain to rein in the new post-truth world and check the spread of fake news. IT Professional. 2019. Vol. 21. №. 4. Pp. 16-24.

13. Zannettou et al., «The Web of False Information: Rumors, FakeNews, Hoaxes, Clickbait, and Various Other Shenanigans» 2019 